\title{
Tn5 transposition in Escherichia coli is repressed by Hfq and activated by over-expression of the small non-coding RNA SgrS
}

\author{
Joseph A Ross, Ryan S Trussler, Morgan D Black, Crystal R McLellan and David B Haniford*
}

\begin{abstract}
Background: Hfq functions in post-transcriptional gene regulation in a wide range of bacteria, usually by promoting base pairing of mRNAs with trans-encoded sRNAs. It was previously shown that Hfq down-regulates Tn10 transposition by inhibiting IS10 transposase expression at the post-transcriptional level. This provided the first example of Hfa playing a role in DNA transposition and led us to ask if a related transposon, $\operatorname{Tn} 5$, is similarly regulated.

Results: We show that Hfq strongly suppresses Tn5 transposition in Escherichia coli by inhibiting 1550 transposase expression. However, in contrast to the situation for Tn10, Hfq primarily inhibits IS50 transposase transcription. As Hfq does not typically function directly in transcription, we searched for a transcription factor that also down-regulated IS5O transposase transcription and is itself under Hfq control. We show that Crp (cyclic AMP receptor protein) fits these criteria as: (1) disruption of the crp gene led to an increase in 1550 transposase expression and the magnitude of this increase was comparable to that observed for an $h f q$ disruption; and (2) Crp expression decreased in $h f q^{-}$. We also demonstrate that IS50 transposase expression and Tn5 transposition are induced by over-expression of the sRNA SgrS and link this response to glucose limitation.
\end{abstract}

Conclusions: Tn5 transposition is negatively regulated by Hfa primarily through inhibition of IS50 transposase transcription. Preliminary results support the possibility that this regulation is mediated through Crp. We also provide evidence that glucose limitation activates 1550 transposase transcription and transposition.

Keywords: Tn5/IS50, Hfq, Crp, SgrS, DNA transposition

\section{Background}

Transposase proteins catalyze the chemical steps in bacterial transposition reactions. It follows that the regulation of expression of these genes is a critical feature in dictating the transposition frequency of most transposons. In many instances, including Tn10/IS10 and Tn5/ IS50, transposase gene promoters are inherently weak. In addition, DNA adenine methylase (DAM) limits initiation of ISIO and IS50 transposase gene transcription by methylating promoter elements $[1,2]$. These factors together make transcription initiation a limiting step in Tn10/IS10 and Tn5/IS50 transposition reactions [3,4]. There are also examples where translation of transposase transcripts is subject to both intrinsic and host levels of

\footnotetext{
* Correspondence: haniford@uwo.ca

Department of Biochemistry, University of Western Ontario, London ONN6A 5C1, Canada
}

regulation. In the case of IS10 transposase, the ribosome binding site is inherently weak and the transposon encodes an antisense RNA that binds the translation initiation region (TIR), blocking ribosome binding [5,6]. There is also evidence that the 'host' protein Hfq helps mediate the pairing interaction between the antisense RNA and the IS10 transposase transcript [7,8].

$\mathrm{Hfq}$ is a global regulator of gene expression in bacteria. It typically functions at the post-transcriptional level, influencing translation initiation and/or transcript stability by catalyzing the pairing of small RNAs (sRNA) and their mRNA targets (Figure 1B and reviewed in [9]). In contrast to the many examples of Hfq acting in a posttranscriptional capacity to impact gene expression, there is (to our knowledge) only one example in the literature of Hfq acting at the level of transcription to influence gene expression. In the case of ribosomal proteins rpsO, rpsT and rpsB-tsf, Hfq was shown to increase transcript 

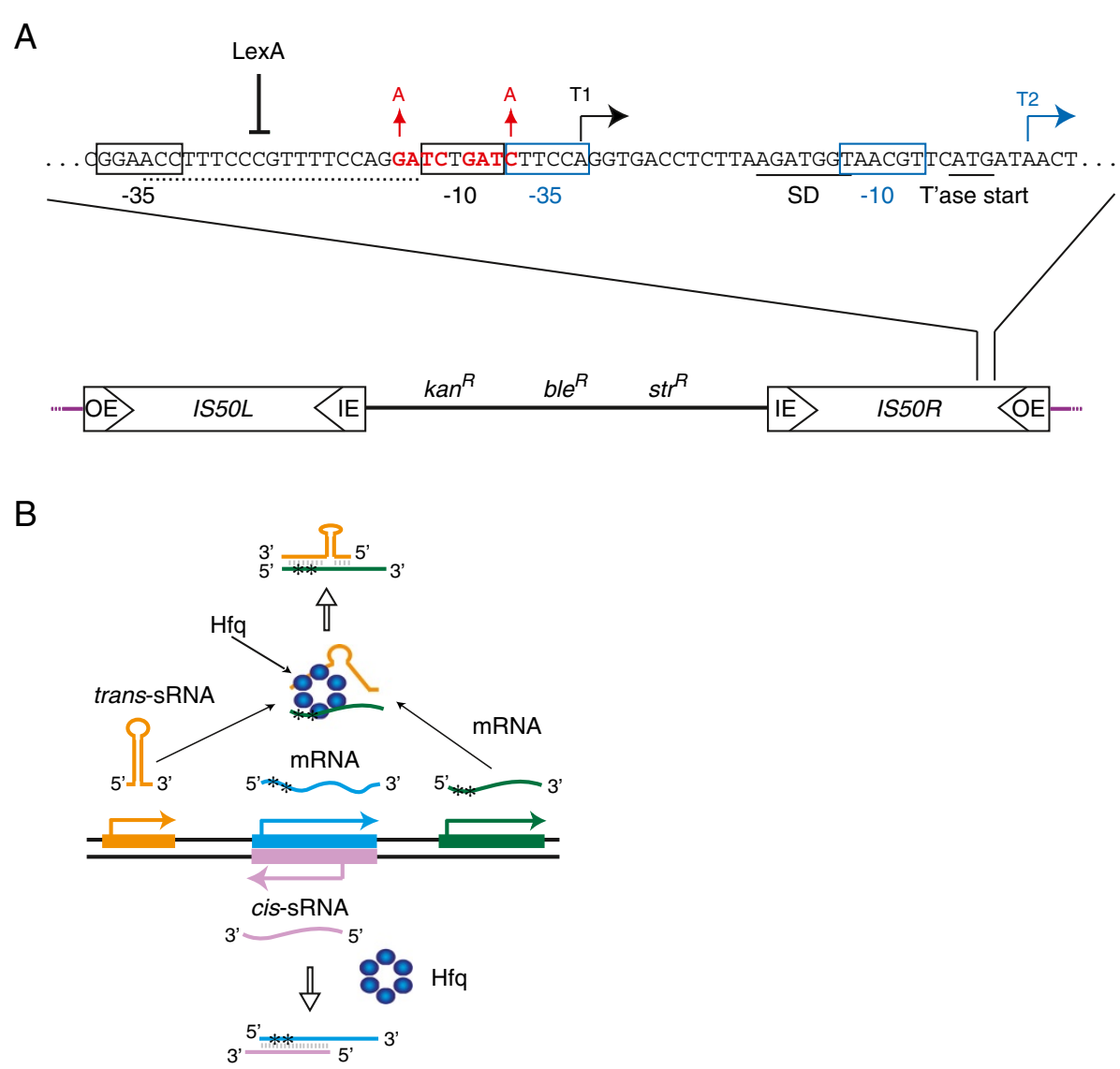

Figure 1 Tn5/IS50 structure and gene expression. (A) The structure of Tn5 is shown along with transcription units within IS50-Right. There are two distinct promoters defined by -35/-10 regions that control transposase (black) and inhibitor (blue) expression. $\mathrm{T1}$ is the transposase transcript and $\mathrm{T} 2$ is the inhibitor transcript. The Shine-Dalgarno sequence of $\mathrm{T} 1$ is also shown. Expression of $\mathrm{T} 1$ but not $\mathrm{T} 2$ is regulated by DAM methylation at two GATC sequences (red) and potentially LexA binding (dotted line defines a putative LexA binding site). Mutations in the dam sites used in this work are shown. $\mathrm{kan}^{R}, \mathrm{ble}^{R}$ and $\mathrm{str}^{R}$ are kanamycin, bleomycin and streptomycin resistance genes, respectively. (B) Post-transcriptional regulation by Hfa. Hfa (blue hexamer) is shown catalyzing the pairing of an SRNA with an mRNA. The sRNA can be either cis or trans encoded relative to its target mRNA. In both cases the sRNA is shown pairing to the translation initiation region of the mRNA (asterisks) and would block translation.

levels without influencing transcript stability. It was suggested that this is accomplished through Hfq binding to secondary structure elements in the respective transcripts that form early in the elongation phase of transcription and that this interaction reduces RNA polymerase pausing [10].

As noted above, Hfq has been implicated in the regulation of Tn10/IS10 transposition. Under conditions of $h f q$ deficiency, a large increase in both Th10/IS10 transposition (up to 80-fold) and transposase expression (up to 7-fold) were observed. The existing evidence is consistent with Hfq acting as a negative regulator of IS1O transposase expression by both antisense dependent and independent pathways. In support of the latter, it was found that $h f q$ deficiency (or $h f q^{-}$) had a significant impact on Tn10 transposition even when the level of antisense RNA was insufficient to impact on transposase expression (that is when $\operatorname{Tn} 10$ is present in single copy in the bacterial chromosome). In addition, there was a synergistic increase in transposase expression when both $h f q$ and the antisense RNA were knocked out, implying that Hfq does not function exclusively in the same pathway as the antisense RNA [7].

Taking the above results into account, and considering that most bacterial transposition systems are not regulated by antisense RNAs, we wondered if Hfq might play a more general role in regulating transposition systems. In the current work, we tested this hypothesis by asking if $\operatorname{Tn} 5$ transposition is also regulated by Hfq. Like $\operatorname{Tn} 10, \operatorname{Tn} 5$ is a composite transposon (Figure 1A). The two transposons are closely related but $T n 5$ lacks an antisense RNA regulatory system and consequently if Hfq were to regulate this system at the post-transcriptional level, it is likely that a trans-encoded sRNA would play a role [11-13]. Tn5 does encode an inhibitor protein that limits Tn5/IS50 transposition by dimerizing with the transposase protein, forming an inactive complex [14]. Transposase and the inhibitor protein are expressed from overlapping promoters, $\mathrm{P} 1$ and 
P2 (color coded in Figure 1A), with the inhibitor transcript (T2) being expressed at a higher level than the transposase transcript (T1). T1 expression is down-regulated by DAM (reviewed in [15]). There is some evidence that P1 is also negatively regulated by LexA, an SOS-inducible transcriptional repressor [16]. However, there is little else known with regard to host proteins that influence either transposase transcription or translation.

In the current work, we show that both $\operatorname{Tn} 5$ transposition and IS5O transposase expression increase significantly in $E$. coli under conditions of $h f q$ deficiency. However, unlike the situation in Tn10/IS10 transposition, the up-regulation of IS50 transposase expression appears mainly to be due to an increase in transposase gene transcription. As Hfq does not typically function directly in transcription, we looked at the possibility that $\mathrm{Hfq}$ regulates IS50 transposase expression by controlling the expression of a transcription factor. Towards this end, we provide evidence that Hfq acts in a regulatory network with $\mathrm{Crp}$ (cyclic AMP receptor protein) to down-regulate IS50 transposase transcription. Finally, we demonstrate that over-expression of an sRNA (SgrS) activates expression of the IS5O transposase gene specifically when cells are grown with glucose as the sole carbon source. Evidence is presented that this up-regulation is a consequence of glucose limitation, demonstrating that the IS50 transposase promoter (and $\operatorname{Tn} 5$ transposition) is responsive to the nutrient status of the cell.

\section{Results}

\section{$\mathrm{Hfq}$ is a potent negative regulator of $\mathrm{Tn} 5$ transposition}

We asked if Hfq regulates Tn5 transposition in E. coli by measuring the frequency of $T n 5$ transposition under conditions of $h f q$ deficiency using the 'mating out' assay. In this assay, an $\mathrm{F}^{+}$donor strain harboring a chromosomal copy of $\operatorname{Tn} 5$ was mated to an $\mathrm{F}^{-}$recipient strain and the mating efficiency and number of transposition events were measured by plating mating mixes on the appropriate selective media (see Methods). We show in Figure 2A that in one donor strain background (DBH179) Tn5 transposition increased by close to 75-fold under conditions of $h f q$ deficiency. Note that we did not have a defective copy of $\operatorname{Tn} 5$ to act as a negative control in this experiment. In lieu of this, we carried out physical mapping on a sampling of colonies present on 'hop' plates to ensure that bona fide transposition events were being measured in both $w t$ and $h f q^{-}$strains (Additional file 1).

We also performed a complementation assay in the DBH179 strain background to further test that the increase in transposition reported above in $h f q^{-}$was actually due to the absence of Hfq, as opposed to possible polar effects of the $h f q$ disruption allele. Towards this end, we introduced $h f q$ on a low-copy plasmid (pDH700) into the $h f q^{-}$strain and measured $\operatorname{Tn} 5$ transposition as above. We observed nearly complete complementation by plasmid- borne $h f q$, as transposition was reduced approximately 45 fold relative to when no $h f q$ was present (Figure 2A). Furthermore, plasmid-encoded variants of $h f q$, including K56A and Y25A, which are impaired for RNA-binding at the 'proximal' and 'distal' surface, respectively, failed to complement $h f q$ deficiency [17]. This confirms that specific functions of $\mathrm{Hfq}$, namely interaction with RNA via known RNA-binding surfaces, are required for effective repression of $\operatorname{Tn} 5$ transposition.

We also tested the impact of $h f q$ deficiency on $\operatorname{Tn} 5$ transposition in a second donor strain background (DBH261) via the 'mating out' assay (Figure $2 \mathrm{~B}$ ). In this experiment $h f q^{-}$also caused an increase in $\operatorname{Tn} 5$ transposition, although the magnitude of the effect was smaller (approximately 9fold) than reported for the DBH179 strain background.

\section{IS50 transposase expression increases in $\mathrm{hfq}^{-}$cells}

We next asked if $h f q$ status influenced IS50 transposase expression. In one approach, we measured transposase expression by constructing IS50-lacZ transcriptional and translational fusions ('TCF' and 'TLF', respectively; see Figure 3A for schematics), integrating these reporters into the chromosome of a $\mathrm{lac}^{-}$E. coli strain (DBH107), and then performing $\beta$-galactosidase assays. This was done for each reporter in isogenic strains that were either $w t, d a m^{-}$or $h f q^{-}$. As expected for a promoter that is DAM-sensitive, transposase expression increased in the context of both transcriptional and translational fusions in the $\mathrm{dam}^{-}$strain relative to $w t$ (approximately19and 25-fold, respectively; Figure 3B). The increase in transposase expression for both constructs in $\mathrm{dam}^{-}$is indicative of expression coming predominantly from the P1 promoter [2]. Transposase expression in TCF and TLF constructs also increased in $h f q^{-}$cells (11-fold and 7.4-fold, respectively), indicating that $\mathrm{Hfq}$ (or a factor under Hfq control) represses IS50 transposase expression. As the TCF encodes only 15 nucleotides of the transposase transcript (T1), it seemed most likely that up-regulation of transposase expression in $h f q^{-}$was primarily due to enhanced transcription in both TCF and TLF constructs.

To further test this possibility, we constructed a TLF reporter (pDH908) wherein the IS50 transposase promoter was replaced by a heterologous promoter (from the lpp gene) whose regulation is not sensitive to $h f q$ status [10]. An isogenic plasmid (pDH795) in which the TLF contained the IS50 transposase promoter was also constructed. Cells ( $w t$ or $h f q^{-}$) were transformed with plasmids containing these constructs and reporter expression was measured as above. The results presented in Figure 4 show that transposase expression increased approximately 9-fold for the construct containing the IS50 promoter and less than 2-fold for the construct 
A

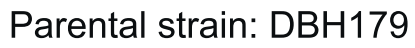

B

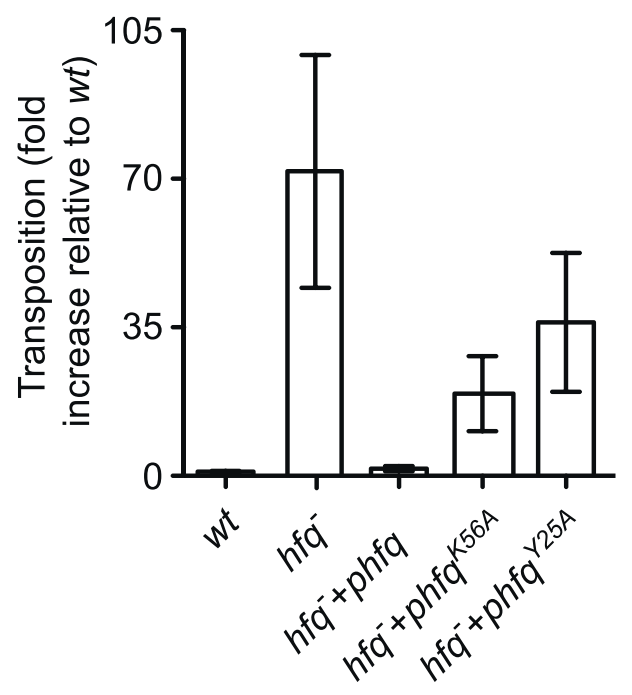

Parental strain: DBH261

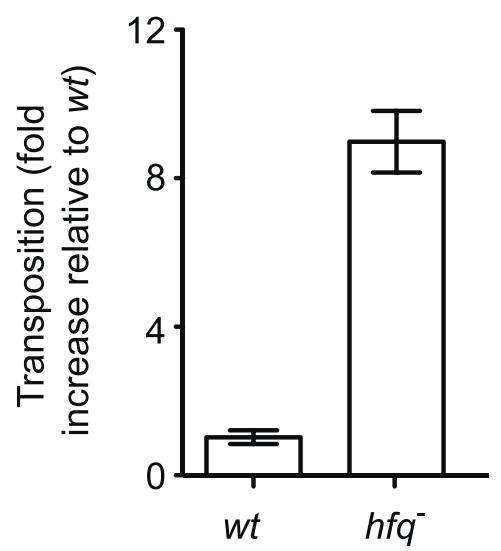

Figure 2 Frequencies of $\operatorname{Tn} 5$ transposition in $\mathbf{h f q}^{-}$versus wt strains of $\boldsymbol{E}$. coli. (A) Th5 transposition from the chromosome of DBH179 and derivatives ( $\mathrm{hfq}^{-}$and $\mathrm{dam}^{-}$) was measured by the conjugal 'mating out' assay as described in Methods. For purposes of trans-complementation, strains contained an empty vector or a low-copy plasmid encoding either wild type $h f q^{W T}$ or mutant forms of $h f q$ (K56A or Y25A) expressed from the $h f q$ P3 promoter. The data was compiled from four independent experiments, each with at least three isolates of each strain. The average transposition frequency was $8.33 \times 10^{-5}$ events per $\mathrm{mL}$ of mating mix for the wt strain (no ' $h f a$ plasmid') and for purposes of comparison this value was set at 1 and all other values normalized to this. The illustration shows the structure of an Hfq hexamer with RNA (gold) bound either to the proximal or distal face [9]. The Y25A mutation inhibits RNA binding to the distal face and the K56A mutation inhibits RNA binding to the proximal face. Adapted from Nature Reviews: Microbiology [9] with permission from Macmillan Publishers. (B) Tn5 transposition from the chromosome of DBH261 and derivatives (hfq and $\mathrm{dam}^{-}$) was measured as in (A). The data shown is from one experiment with five independent isolates of each strain. The average transposition frequency for the wt strain was $2.57 \times 10^{-6}$ events per $\mathrm{mL}$ of mating mix. In (A) and (B) the error bars indicate standard error of the mean.

containing the lpp promoter. These results support our contention that Hfq-directed regulation of IS5O transposase expression occurs at the transcriptional level because the absence of the IS50 promoter and not the presence of the IS50 5' UTR was the dominant factor in observing strong up-regulation of reporter expression under conditions of $h f q$ deficiency.
Hfq impacts steady-state levels of full-length IS50 transposase mRNA

To further assess the impact of $h f q$ deficiency on transposase gene expression, we looked at both the steadystate level and the stability of the transposase transcript (T1) in $h f q^{+}$and $h f q^{-}$cells. For the steady-state analysis, total RNA was isolated from various strains ( $w t$ or $h f q^{-}$) 


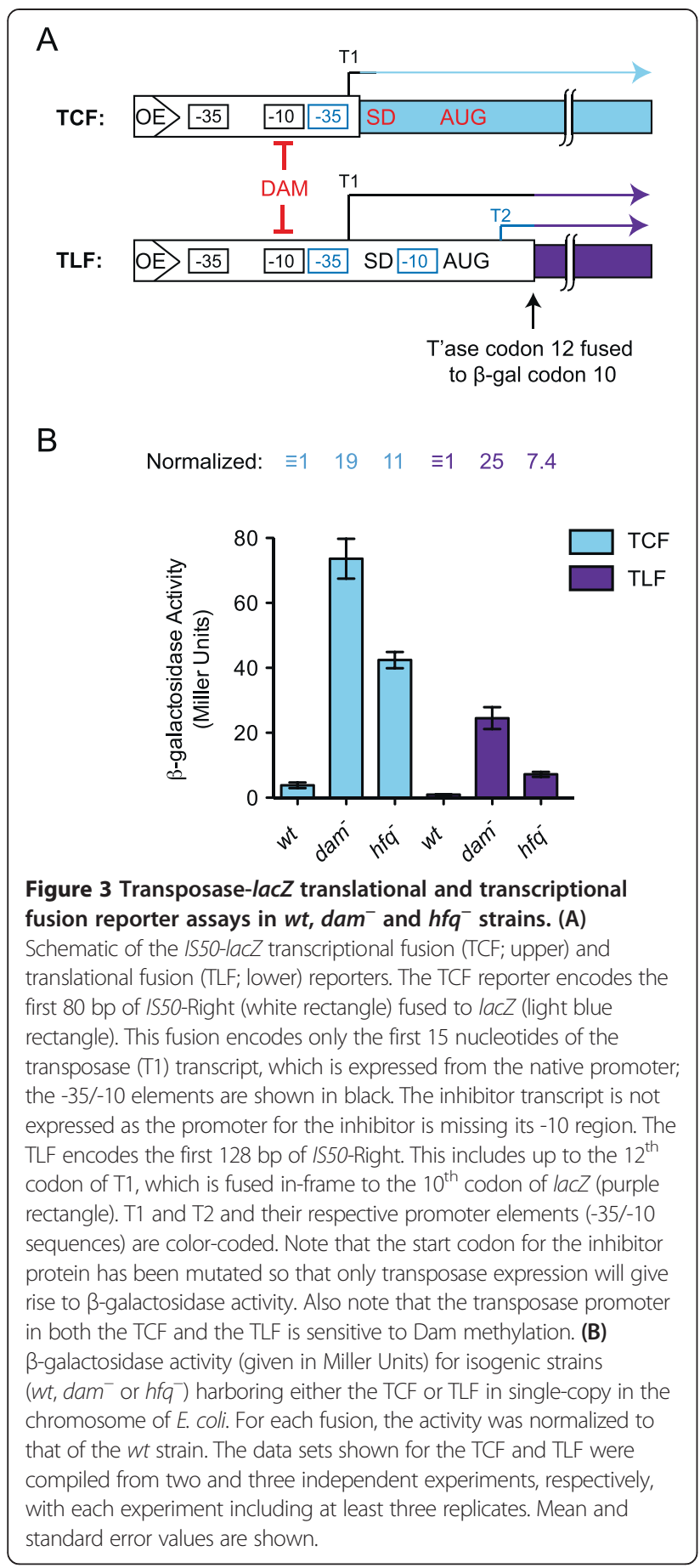

(DBH33 background) containing a multi-copy plasmid encoding the full-length transposase gene under the control of its native promoter. In addition to the $w t$ version of this plasmid (pDH533), we also analyzed a mutant form containing mutations in the overlapping dam methylation sites in the transposase promoter (pDH752) (see Figure 1A); these mutations make this construct DAM insensitive. Primer extension was used to detect both $\mathrm{T} 1$ and $\mathrm{T} 2$ transcripts, as well as the lpp transcript

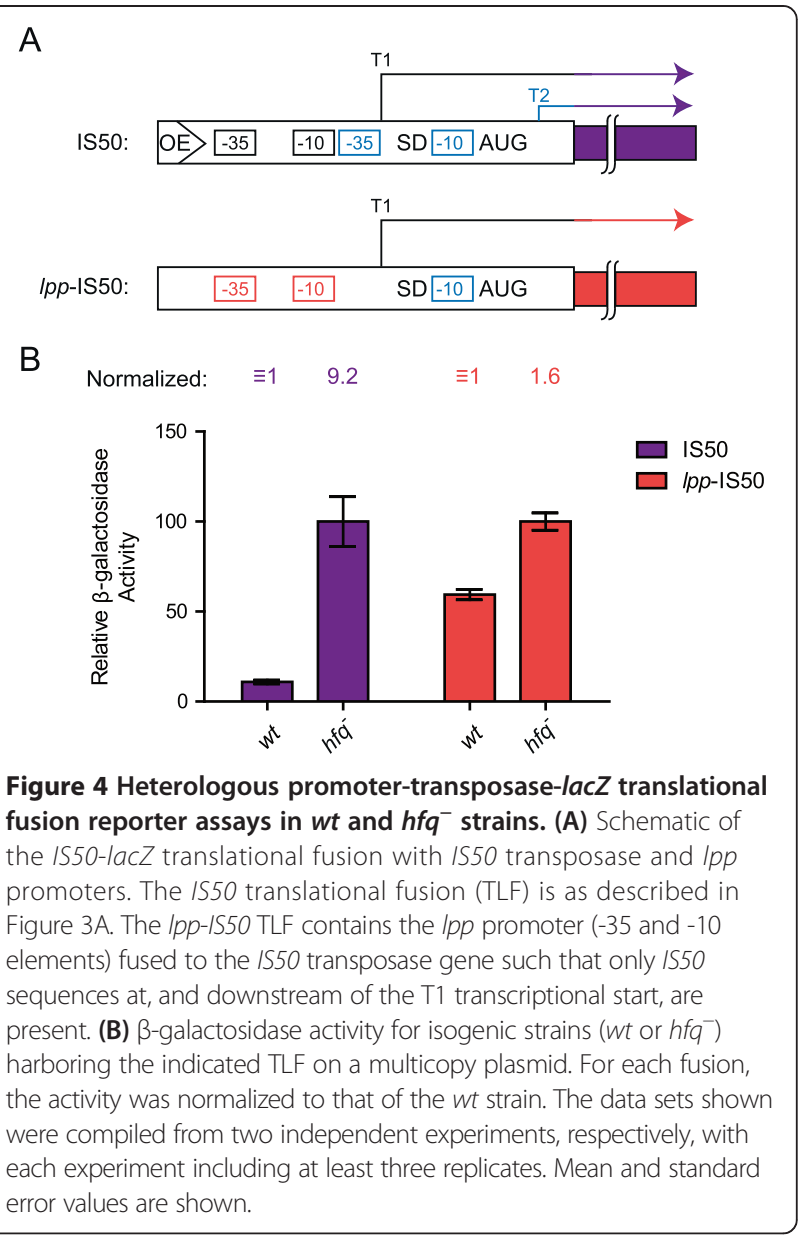

(loading control). As expected for a dam-sensitive promoter, levels of $\mathrm{T} 1$ increased substantially (approximately 8 -fold) in $w t$ cells containing the plasmid with the daminsensitive promoter versus $w t$ cells containing the $w t$ promoter (compare lanes 3 to 7 with lanes 8 to 12 in Figure 5A and bar graph in Figure 5B). In contrast, there was no significant change in T2 levels in the above samples. In $h f q^{-}$ (wt promoter) there was also a substantial increase in $\mathrm{T} 1$ levels (11-fold) versus the $w t$ strain (compare lanes 3 to 7 with lanes 14 to 18) and no significant change in T2 levels. Thus in an $h f q^{-}$background there was an increase in the steady-state level of transposase transcript and this increase was slightly greater than that observed when methylation of the transposase promoter was blocked.

We also looked at the combined impact of knocking out Hfq and blocking DAM methylation on T1 levels (lanes 19 to 23 in Figure 5A). In comparison to $w t$, the 'double mutant' situation resulted in a 45 -fold increase in T1 levels. Based on the observed synergy, we think it unlikely that the observed impact of deleting $h f q$ is linked to the regulation of dam expression.

To directly test if a component of Hfq-directed repression of IS50 transposase expression is post-transcriptional, 


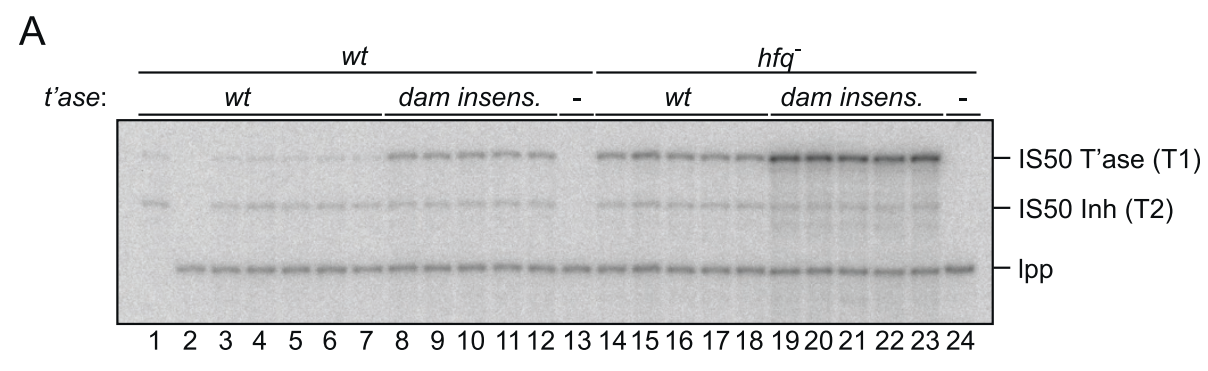

B

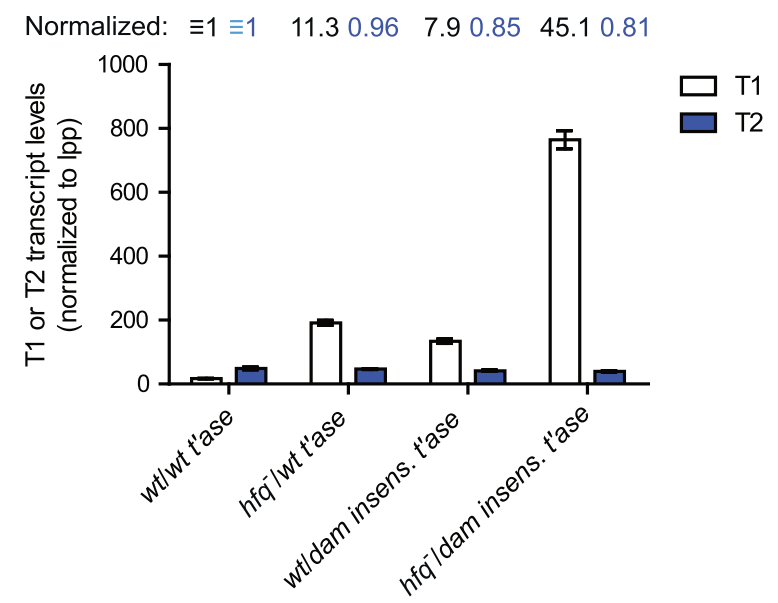

Figure 5 Steady-state levels of IS50 transposase mRNA in wt and $\mathbf{h f q}^{-}$cells. (A) Plasmids encoding wt or DAM-Insensitive IS50 transposase genes were transformed into wt (DBH33) or $\mathrm{hfq}^{-}$(DBH16) E. coli strains. Total RNA was isolated from five different clones grown to mid-log phase for each of the indicated strains. Primer extension reactions were multiplexed using ${ }^{32} \mathrm{P}$-labeled primers complimentary to IS50 transposase (primer $\mathrm{oDH} 230$ ) and Ipp (primer oDH390) RNAs. The corresponding CDNAs were analyzed on a 10\% sequencing gel. T1 and T2 are defined in Figure 1. Note that transcription of lpp is known to be insensitive to hfa status [10]. (B) Summary of data in (A).

we compared the stability of the IS50 transposase mRNA (T1) in isogenic wt and $h f q^{-}$strains. Total RNA was isolated from a pair of rifampicin-sensitive strains (TM338 and TM618) containing a plasmid encoding IS50 transposase (pDH533) before and after rifampicin treatment as shown in Figure 6. Transposase mRNA was detected by primer extension. In the $h f q^{-}$strain the half-life of the T1 transcript increased by approximately 1.7 -fold, revealing that $h f q$ status does impact on transposase mRNA stability.

Taken together, the results from Figures 3, 4, 5 and 6 show that IS50 transposase expression is substantially reduced in an $h f q^{+}$relative to an $h f q^{-}$strain and that $h f q$ status primarily affects transposase transcription.

\section{Regulation of $\operatorname{Tn} 5$ transposase expression by global transcriptional regulators}

As Hfq does not typically function directly in transcription, we set out to define a transcription factor that downregulates $I S 50$ transposase transcription and is itself regulated by Hfq. Toward this end, we asked if disrupting genes for two global transcription factors, Crp and Lrp [18], had an impact on IS50 transposase expression. Note that we had to construct new TCF reporter strains for this work because the available crp and lrp disruption strains we used to transduce DBH107 to either $\mathrm{crp}^{-}$or $\operatorname{lrp} \mathrm{p}^{-}$ were marked with the same antibiotic resistance gene used to select for a lysogen with a chromosomal copy of the TCF. We show in Figure 7A that $c r p^{-}$but not $l r p^{-}$had a substantial impact on transposase expression. For example, in cells grown in exponential phase in Luria broth (LB), there was up-regulation of transposase expression (approximately 4-fold) in both $\mathrm{crp}^{-}$(DBH307) and $h f q^{-}$ (DBH306) strains but not in the lrp $^{-}$strain (DBH315). We also performed semi-quantitative RT-PCR and show that transposase-lac $Z$ transcript levels increased similarly in $c r p^{-}$and $h f q^{-}$strains (Figure 7B). These results are consistent with Crp being a negative regulator of IS50 transposase transcription.

We next asked if Crp expression was regulated by Hfq. Notably, work done in Yersinia pestis has shown that Hfq positively regulates Crp expression at the posttranscriptional level [20]. Towards this end we performed Western blot analysis with a Crp antibody on E. coli cell extracts from $w t$ (DBH303), $h f q^{-}$(DBH306) and $c r p^{-}$ (DBH307) strains (Figure 7C). The results show that lower levels of Crp are present in the $h f q^{-}$strain, which is consistent with $\mathrm{Hfq}$ also being a positive regulator of Crp expression in E. coli. 


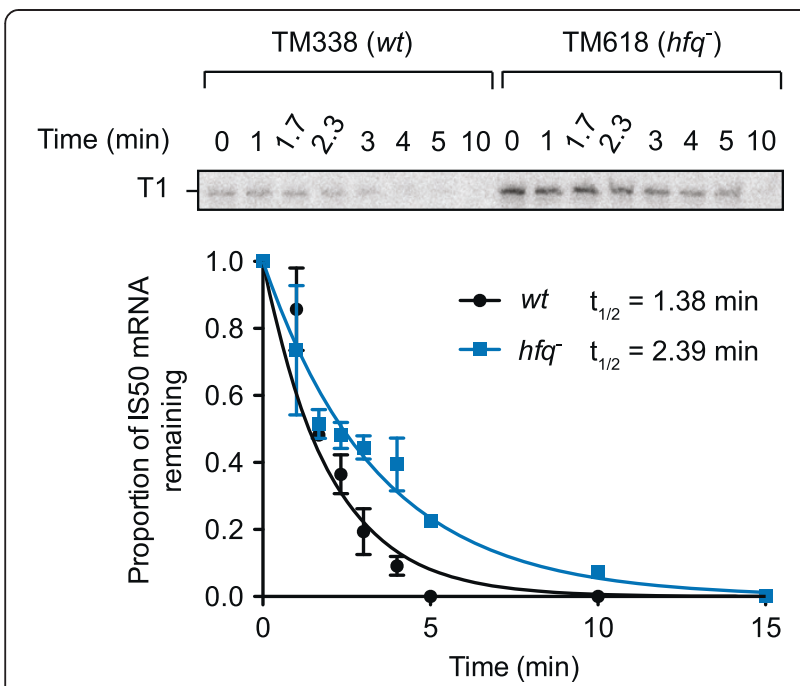

Figure 6 IS50 transposase mRNA half-life analysis. Strains TM338 (wt) and TM618 ( $\left.\mathrm{hfq}^{-}\right)$were transformed with 1550 transposase encoding plasmid pDH533 and total RNA was isolated either before or after the addition of rifampicin (at the indicated time points). Transposase RNA was detected as described in Figure 5. The bands were quantified (ImageQuant) and T1 normalized to un-extended primer before plotting the proportion of RNA remaining after rifampicin addition (time zero $=1.0$ ). The data was fit to a onephase exponential decay curve by non-linear regression (Prism) to determine the half-life $\left(t_{1 / 2}\right)$. The data shown is a compilation from two independent experiments.

Finally, we assessed the impact of knocking out crp on Tn5 transposition frequency using the 'mating out' assay (Figure 7D). In the absence of $c r p, T n 5$ transposition increased 7-fold, which is consistent with results from the transposase expression experiments.

\section{IS50 transposase expression and $\operatorname{Tn} 5$ transposition are up-regulated by over-expression of the sRNA SgrS}

Over-expression of sRNAs can alter Hfq-regulated networks by limiting the availability of $\mathrm{Hfq}[21,22]$. Given our findings that $\operatorname{Tn} 5$ transposition and transposase gene expression are affected by $h f q$ status, we asked if IS5O transposase expression might be sensitive to Hfq-titration. Towards this end, we measured transposase expression from the TLF under conditions where a single sRNA was over-expressed from an IPTG inducible promoter (pLlacO) in DBH33, which is $l a c I^{q}$. Our initial screen included four different Hfq-dependent sRNAs, including RybB, RyeB, $\mathrm{MicC}$ and SgrS, all of which are expected to tightly bind Hfq in vivo; apparent equilibrium dissociation constants of approximately $3.3 \mathrm{nM}$ and $<20 \mathrm{nM}$ have been measured for MicC and SgrS, respectively [23-25]. Cells were grown in M9 glucose and sRNA expression was induced for 4 hours in exponential phase. We show in Figure 8A that only one of the sRNAs tested, SgrS, had a significant impact on transposase expression. Induction of SgrS increased transposase expression just over three-fold. Given the comparable Hfq binding affinities of the sRNAs tested, it seemed unlikely that SgrS expression was increasing transposase expression through an Hfq-titration mechanism.

SgrS down-regulates the expression of several known targets, including the primary glucose transporter encoded by the $p t s G$ gene, a mannose transporter encoded by man$X Y Z$ and it up-regulates the expression of $y i g L$, a phosphatase involved in phospho-sugar detoxification [26]. As we observed up-regulation of IS50 transposase expression in cells over-expressing SgrS in M9 glucose media, we considered the possibility that this effect was a response to glucose limitation. In fact, we show in Additional file 2 that induction of SgrS in M9 glucose resulted in a substantial slowing of bacterial growth, as would be expected if nutrients had become growth-rate limiting. To further test the glucose limitation hypothesis, we performed a similar experiment in rich media (LB) and in M9 glucose supplemented with glycerol, a carbon source whose import is not dependent on glucose transporters [27]. We also tested the response of the reporter to over-expression of an SgrS mutant, sgrS1, that is incapable of downregulating glucose import [28]. In these experiments we used a Tn5 TCF as a reporter in the DBH107 strain background; DBH107 has a complete deletion of the lac operon and consequently the plasmid-encoded sRNA genes are constitutively expressed. To avoid problems in growing these cells, cultures were initially propagated in either LB or M9 glucose/glycerol and then where indicated, switched to other media.

We show in Figure 8B that after approximately 4 hours of SgrS over-expression in M9 glucose, reporter expression increased close to 5 -fold relative to a 'vector' control. In contrast, over-expression of SgrS1 was incapable of up-regulating reporter expression under these same conditions, suggesting that SgrS must be able to downregulate glucose import and or retention in order to increase transposase transcription. When cells were grown in M9 glucose supplemented with glycerol, expression of SgrS as above caused only an approximately 2 -fold increase in transposase expression. Importantly, the reduced effects of SgrS on transposase expression under 'glycerol' conditions cannot be explained by differential expression of the respective sRNAs, as levels of SgrS and SgrS1 were similar in M9 glucose with or without glycerol (Figure 8C). Also, we failed to see significant transposase induction when SgrS was over-expressed in LB media where there are multiple carbon sources. Finally, consistent with the glucose limitation hypothesis, we also show in Figure $8 \mathrm{~B}$ that increased transposase expression resulting from SgrS expression in M9 glucose was the only condition that inhibited cell growth.

Given that transposition frequency is expected to be roughly proportional to transposase expression, we also asked if glucose limitation had an impact on $\operatorname{Tn} 5$ 

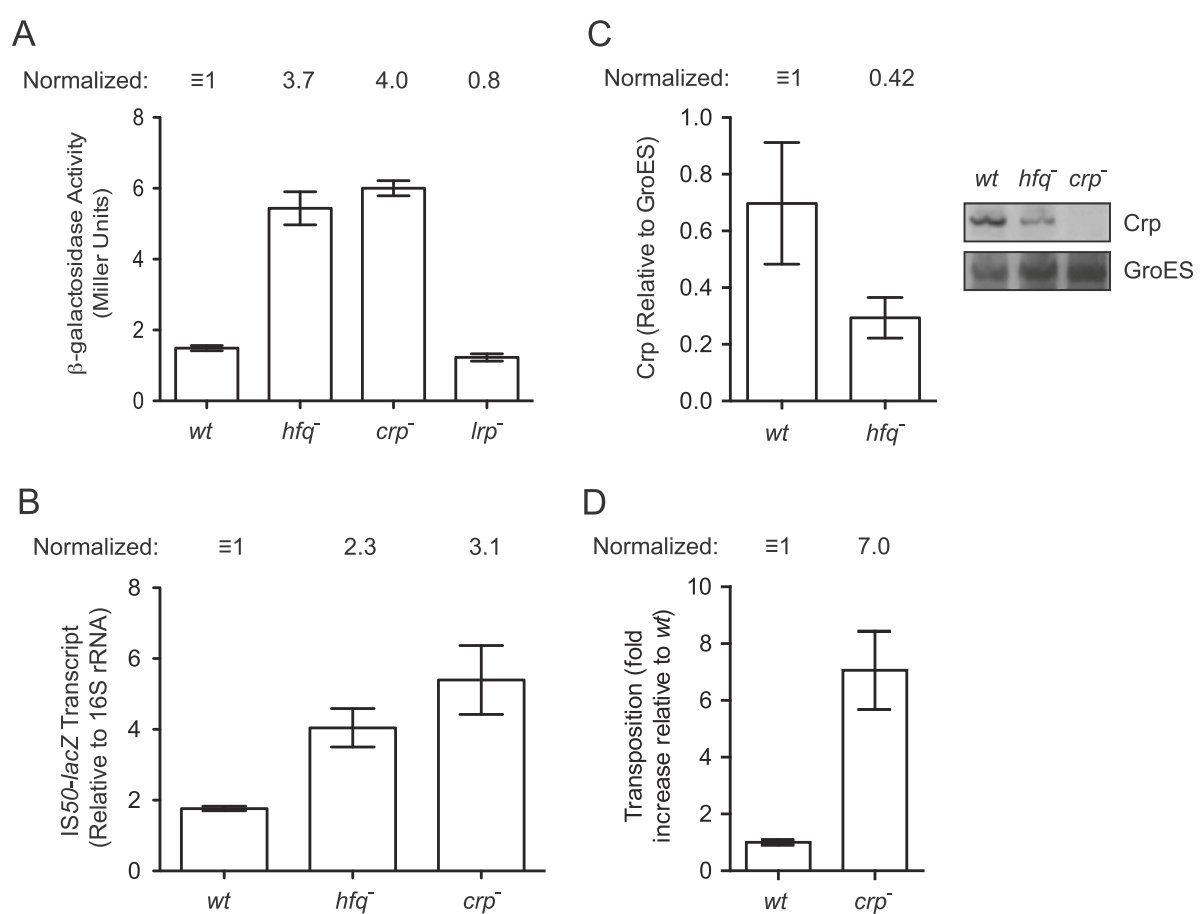

Figure 7 Gene expression and $\operatorname{Tn} 5$ transposition assays in strains harboring disruptions of global transcriptional regulators. (A) $\beta$-galactosidase activity for isogenic strains ( $w t, h f q^{-}, c r p^{-}$and $/ r p^{-}$) harboring the TCF in single-copy in the chromosome (DBH303 and derivatives). Cells were grown to mid-log phase in Luria broth (LB). Mean and standard error values of duplicate experiments, each of which included at least three replicates, are shown. (B) IS50-lacZ transcript levels. Total RNA was extracted from cells described in panel (A), and subjected to RT-PCR. (C) Western blot analysis of Crp levels in cellular extracts from wt and $h f^{-}{ }^{-}$cells grown in LB. As a negative control, crp ${ }^{-}$cells were also analyzed. A representative image is shown in the inset. Crp levels were normalized to GroES, which is known to be insensitive to hfa status [19]. (D) Tn5 transposition from the chromosome of DBH179 (wt) and DBH345 ( $\mathrm{crp}^{-}$) was measured by the conjugal 'mating out' assay as described in Methods. The data is from a single experiment wherein five independent clones of each strain were tested. Mean and standard error values are shown. The average transposition frequency was $1.70 \times 10^{-4}$ events per $\mathrm{mL}$ of mating mix for the wt strain and for purposes of comparison this value was set at 1 and the ' $c r p$ ' value was normalized to this. In two other independent experiments the fold increase in $\operatorname{Tn} 5$ transposition for $\mathrm{crp}^{-}$versus wt did not differ by more than 20\% compared to the experiment shown (data not shown). For experiments in (A-C), mean and standard error values from at least three independent isolates are shown.

transposition. Cells encoding a chromosomal copy of $\operatorname{Tn} 5$ were transformed with an SgrS-expressing plasmid (or vector only control) and the frequency of Tn5 transposition was measured using the 'mating out' assay. Note that cells were grown in M9 glucose media and SgrS expression was induced only when donor strains were subcultured on the day of mating. We show in Figure 9 that induction specifically of SgrS resulted in a 5-fold increase in Tn5 transposition relative to the vector only control. Notably, when cells were grown in M9 supplemented with glucose and glycerol, induction of SgrS did not result in a significant increase in $\operatorname{Tn} 5$ transposition. Also, we observed a reduced growth rate only in cultures where SgrS was induced in M9 glucose media (data not shown). The results of the 'mating out' analysis are thus entirely consistent with the gene expression experiments presented in Figure 8.

\section{Discussion}

$\mathrm{Hfq}$ is a global regulator of gene expression in bacteria. However, until recently, Hfq had not been linked to the control of transposable elements. Work in the Tn10/ IS10 system provided the first example of $\mathrm{Hfq}$ inhibiting a transposon [7]. In the current work, we asked if the transposition of a related element, $\operatorname{Tn} 5 / I S 50$, is also regulated by Hfq. We show that Tn5 transposition and IS50 transposase expression are repressed by Hfq; however, the mechanism of repression is atypical for Hfq, involving predominantly a block in IS50 transposase transcription. Preliminary evidence is presented that is consistent with Hfq modulating IS5O transposase transcription through regulation of Crp. We also show that transposase transcription and Tn 5 transposition are activated by overexpression of the sRNA SgrS and provide evidence that this is a transcriptional response to glucose limitation.

\section{Hfq negatively regulates $\mathrm{Tn} 5$ transposition}

The results of 'mating out' experiments were consistent with $\mathrm{Hfq}$ acting as a strong negative regulator of $\operatorname{Tn} 5$ transposition. $\operatorname{Tn} 5$ transposition increased close to 75 -fold 


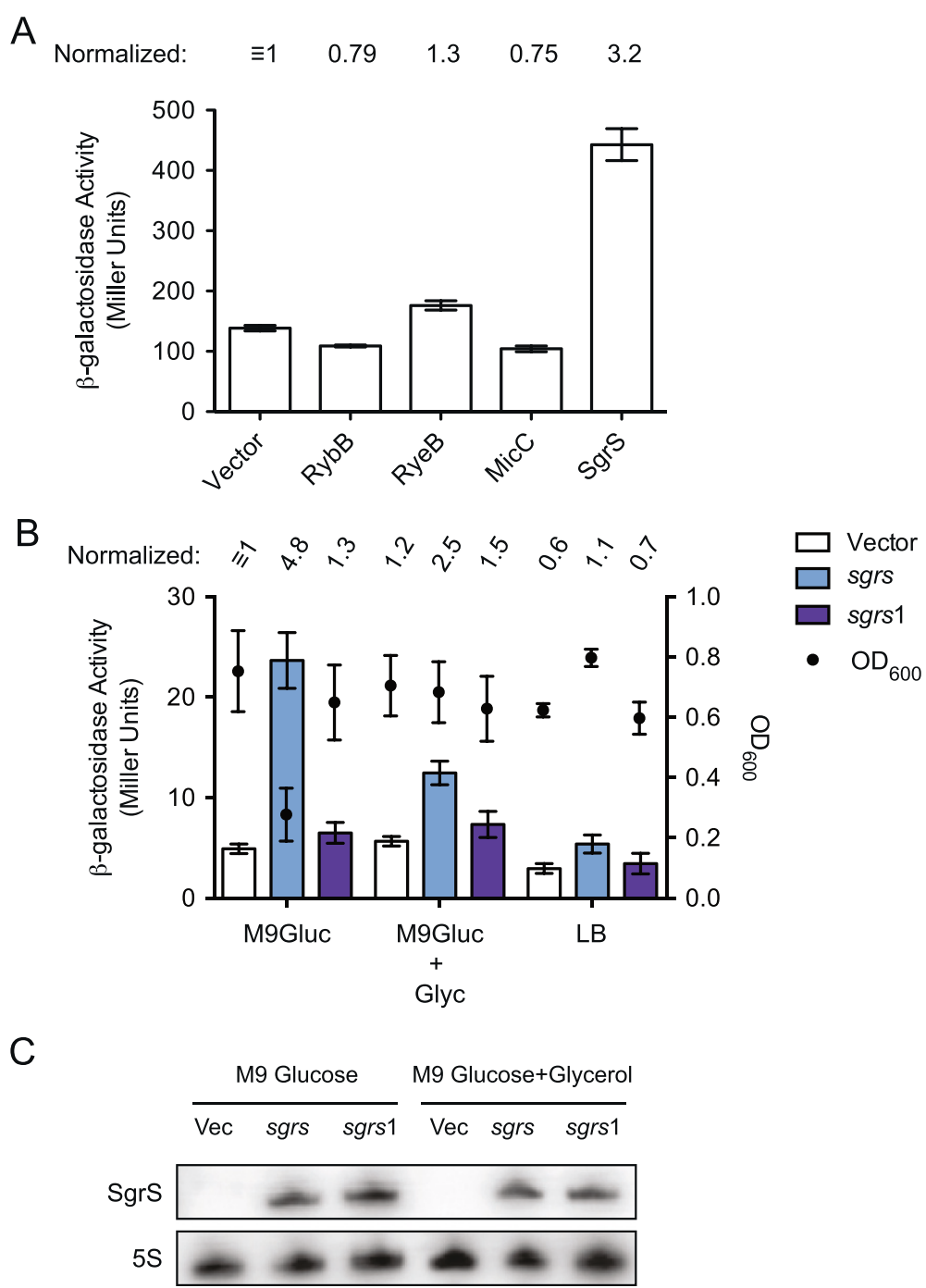

Figure 8 Transposase-lacZ expression assays in cells over-expressing sRNAs. (A) Transposase expression from an IS50 translational fusion (TLF) (see Figure 3A) present on a low-copy plasmid (pDH798) was measured in the presence of a compatible plasmid expressing one of the indicated sRNAs from the inducible pLlacO promoter in DBH33. Cells were grown in M9 glucose and $0.1 \mathrm{mM}$ IPTG was added to subcultures to induce sRNA expression. Transposase expression was measured 4 hours after IPTG addition. Expression levels were normalized to the strain with the vector only control. (B) The impact of different growth media on SgrS-induced up-regulation of transposase expression was evaluated using a single-copy TCF fusion (see Figure 3A) present in the chromosome of DBH265. Note that the sgrS1 allele of SgrS contains a two-nucleotide mutation that inhibits its ability to down-regulate expression of the glucose transporter encoded by pts G. Subcultures were grown in either M9 glucose, M9 glucose + glycerol, or Luria broth (LB), as indicated. $\beta$-galactosidase activity was measured approximately 4 to 6 hours after subcultures were started. In (A) and (B) mean and standard error values of duplicate experiments, each of which included at least three replicates, are shown. (C) Northern blot of RNA isolated from cells in (B). RNA was extracted from cells immediately before starting the Miller assay and visualized by Northern blotting with ${ }^{32}$ P-labeled RNA probes complementary to either SgrS or the 5S rRNA (internal control).

in one $h f q^{-}$strain (DBH179 background). The magnitude of this increase was somewhat surprising given that upregulation of $T n 10$ in $h f q^{-}$, under essentially antisenseminus conditions, was about 7-fold [7]. However, in a different $h f q^{-}$strain (DBH261 background) Tn5 transposition increased only 9 -fold. At this point it is unclear why there was such a large discrepancy in the 'mating out' values for the two strains. One possibility is that colony counts in the DBH179 'mating out' $\left(h f q^{-}\right)$included clones that had 'jack-pot' events. That is, colonies were counted that did not derive from independent transposition events. This could explain the high standard error associated with the transposition frequency in the $h f q^{-}$column in Figure 2A. If, for example, we removed the 3 most prominent outliers from the (DBH179) $h f q^{-}$data set, the fold increase in transposition dropped to 15 -fold, which is more in line with what we observed in the DBH261 strain background and for $\operatorname{Tn} 10$ in single copy [7]. 


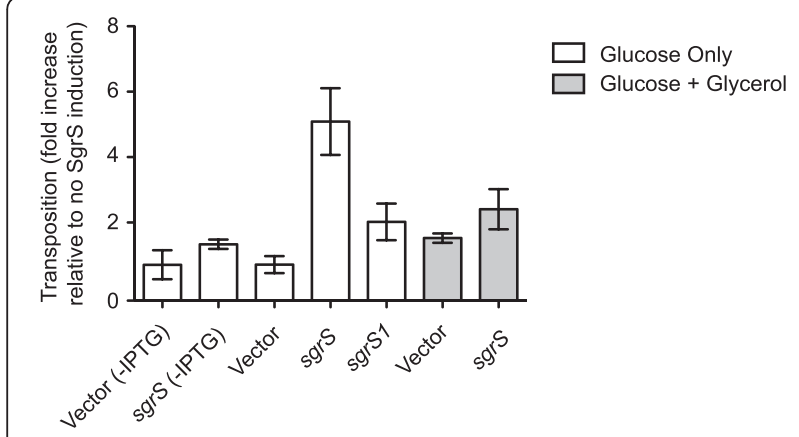

Figure 9 Impact of SgrS expression on Tn5 transposition. Transposition of a chromosomal copy of Tn5 was measured in DBH179 using the 'mating out' assay. DBH179 containing one of the indicated plasmids was grown overnight in M9 glucose and then subcultured in either M9 glucose or M9 glucose plus glycerol before mating with the recipient strain and plating on selective media as described in Figure 2. IPTG was added to the subculture (to $0.1 \mathrm{mM}$ ) to induce SgrS expression, except where indicated (-IPTG). The average transposition frequency for the 'no SgrS' control was $5.52 \times 10^{-5}$ events per $\mathrm{mL}$ of mating mix. All other transposition frequencies were normalized to this value. Mean and standard error values of duplicate experiments, each of which included at least five replicates for each experimental group, are shown.

A trans-complementation (Figure 2A) experiment provided definitive proof that the increase in $\operatorname{Tn} 5$ transposition detected in one of our $h f q^{-}$'mating out' strains (DBH179 background) was in fact due to $h f q$ deficiency. In addition, the failure of two Hfq RNA-binding face mutants to provide complementation was consistent with Hfq-directed inhibition of Tn5 transposition relying on functions of Hfq required in canonical Hfq-directed regulatory pathways [17]. That is, Hfq must retain the ability to bind both mRNAs and sRNAs to influence Tn5 transposition.

\section{$\mathrm{Hfq}, \mathrm{Crp}$ and IS50 transposase gene expression}

Evidence that $h f q$ status influences IS50 transposase expression came from two types of experiments. First, the expression of transposase-lac $Z$ reporter genes in both transcriptional and translational fusion constructs increased significantly under conditions of $h f q$ deficiency. Second, the steady-state level of the native transposase transcript also increased significantly in $h f q^{-}$. Importantly, the large increase in steady-state transcript level (11-fold) coincided with a less substantial increase in transposase mRNA stability (less than 2-fold increase in half-life). In addition, up-regulation of reporter expression in $h f q^{-}$for a TLF was almost completely abrogated when the IS50 transposase promoter was replaced by a heterologous promoter. Taken together, these results are consistent with Hfq (or a factor regulated by Hfq) suppressing IS50 transposase expression predominantly at the level of transcription. Notably the suppressive effect of Hfq on IS5O transposase transcription was remarkably specific, as the level of a second transcript (T2) encoded by $I S 50$ was not affected by $h f q$ status.

As Hfq does not typically act directly in gene transcription, we think it likely that Hfq acts indirectly on the IS50 transposase promoter. In addition to DAM, only one other transcription factor, LexA, has been implicated as a regulator of transposase transcription. There is a weak LexAbinding site in the transposase promoter (Figure 1A); however, lexA deficiency was shown to increase transposase transcription only two to three-fold in a TCF [16]. As we have seen increases in transposase expression of up to 11fold for a TCF in $h f q^{-}$, it seems unlikely that Hfq would be working through LexA. In contrast, transposase expression increased in $\mathrm{dam}^{-}$to a level more in line with that observed in $h f q^{-}$(less than two-fold difference in the TCF). However, the observed synergy between $h f q^{-}$and mutations that rendered the IS50 transposase promoter DAMinsensitive led us to conclude that $\mathrm{Hfq}$ does not regulate IS50 transcription by impacting DAM levels (and, therefore, promoter methylation). These results provided motivation to search for other targets of Hfq that impinge on IS50 transposase transcription. This search identified Crp as an additional negative regulator of IS50 transposase transcription. Notably, transposase expression increased to approximately the same level in $\mathrm{crp}^{-}$and $h f q^{-}$in the experiment in Figure 7. The similar magnitude of upregulation of transposase expression in $h f q^{-}$and $c r p^{-}$could be indicative of $\mathrm{Hfq}$ acting upstream of Crp to inhibit transposase expression. We did in fact find evidence of Hfq positively regulating Crp protein levels (Figure 7C). This observation is consistent with work recently published in the $Y$. pestis system where it was found that Crp protein levels decreased approximately five-fold in an $h f q$ disruption strain [20].

Crp is a known activator/repressor of transcription [18] and, therefore, more likely than Hfq to be directly involved in regulating IS50 transposase expression at the transcriptional level. Given our evidence that Hfq positively regulates crp expression, a plausible scenario explaining our expression data is that the observed up-regulation of IS50 transposase transcription in $h f q^{-}$is a result of decreased Crp protein levels. Crp may act either directly or indirectly on the IS5O transposase promoter to repress transcription. This is currently a working model as we have not yet tested the possibility that Crp binds the IS50 transposase promoter and it may only be coincidental that transposase expression increased to similar levels in $h f q^{-}$and $c r p^{-}$strains. Notably, we also found that Tn5 transposition increased when the crp gene was disrupted, although the extent of the increase was smaller than that observed in the isogenic $h f q$ disruption strain. This could be indicative of additional factors in the $\mathrm{Hfq}$ regulon impinging on $\operatorname{Tn} 5$ transposition.

There is precedent for Crp down-regulating the transcription of a transposase gene. In the case of IS2, 
transposase transcription increased close to 200-fold in $\mathrm{crp}^{-}$. It was also shown through protein-DNA footprinting that Crp binds directly to the IS2 transposase promoter [29]. Interestingly, based on the consensus binding sequence for Crp, the authors of the above study predicted that Crp would bind to the IS50 transposase gene. However, the predicted crp binding site is located downstream of the transposase promoter and is not present in our TCF (where we detected increased transposase expression in $\mathrm{crp}^{-}$). Nevertheless, it would be worthwhile to test for Crp binding to the IS50 transposase promoter as the results of Crp ChIP-chip studies revealed the presence of thousands of weak crp binding sites scattered throughout the E. coli genome [30]. It is also possible that Crp acts indirectly on the IS50 transposase promoter by regulating the expression of another transcription factor.

\section{Tn5 transposition and metabolic stress}

We also identified conditions that activate transposase expression and transposition; over-expression of the sRNA SgrS increased transposase expression and transposition approximately five-fold. We favor the possibility that this induction is a consequence of glucose limitation but cannot formally rule out the possibility that SgrS targets an as yet undefined regulatory pathway that impinges on transposase expression. Our reasoning for this is that we observed induction of transposase expression and transposition specifically when cells were grown with glucose as the major carbon source and SgrS is known to prevent expression and function of the major glucose transporter encoded by the ptsG gene [26]. Consistent with this idea, we found that transposase induction levels correlated with a reduced growth rate. Furthermore, we demonstrated that: (i) an allele of SgrS (sgrS1) that is incapable of down-regulating $p t s G$ expression failed to induce transposase expression in M9 glucose; (ii) under conditions where SgrS was expressed in M9 glucose media supplemented with glycerol, we failed to see induction of transposase expression to the same extent as when glycerol was absent; (iii) SgrS expression did not impact transposase expression when cells were grown in rich media (LB) and (iv) over-expression of 3 other sRNAs (RybB, RyeB and MicC) that are not expected to influence glucose transport did not increase transposase expression in M9 glucose [31-33]. Precedent for nutritional stress influencing transposition comes from earlier work in the IS903 system where mutations in a gene $(\operatorname{asp} A)$ required for fermentative metabolism during anaerobic growth caused transposition to occur at an accelerated rate [34].

At this point it is unclear as to what factors are driving the induction of the transposase gene under SgrS overexpression conditions. With regard to further defining the mechanism of IS5O transposase up-regulation under
SgrS over-expression conditions, it would also be advantageous to find alternative experimental conditions for achieving this increased expression. If, for example, simply starving cells by restricting a carbon source during growth achieves the same end as over-expressing SgrS in M9 glucose media, an unbiased screen to search for genetic factors that are necessary for the up-regulation of transposase expression could be performed to reveal the regulatory network impinging on the transposase promoter. As it stands, any factors that influence SgrS expression would interfere with the outcome of such a screen. Alternatively, if it was found that restricting glucose is not sufficient for inducing transposase expression, the possibility that SgrS plays a more direct role in controlling transposase expression would have to be considered.

\section{Conclusions}

In this work, we have identified several genes that impact on IS50 transposase expression, including $h f q$, crp and sgrS. Hfq and Crp proteins are negative regulators and SgrS RNA (under specific growth conditions) is a positive regulator of transposase gene expression. Exactly how these factors impinge on transposase expression remains to be worked out and at this point it is not clear if we are seeing modulation of the same regulatory network in opposite directions when $h f q$ and crp genes are disrupted and SgrS RNA is over-expressed. Tn5/IS50 is the second transposon identified that is affected by disruption of the $h f q$ gene and the first that does not encode an antisense RNA. This raises the possibility that $\mathrm{Hfq}$ influences the transposition frequency of many other bacterial transposons.

\section{Methods}

\section{Plasmids, bacteriophage and strains}

The IS50 translational fusion plasmid (pDH798) is a pWKS30-derivative containing base pairs 1 to 431 of $I S 50$ (nucleotides 1 to 366 of T1) fused to codon 10 [35] of the E. coli lacZ gene. The IS50 transcriptional fusion plasmid (pDH682) is a pUC18-derivative containing base pairs 1 to 80 of $I S 50$ (nucleotides 1 to 15 of T1) fused to nucleotide -16 (relative to the translational start codon) of lacZ. Plasmids encoding sRNAs (pDH764, sgrS; pDH766, rybB; $\mathrm{pDH768}$, micC; pDH772, ryeB) and the corresponding empty vector control ( $\mathrm{pDH} 763$ ) were kindly provided by $\mathrm{S}$ Gottesman. The plasmid encoding sgrS1 (pDH895) was kindly provided by $\mathrm{C}$ Vanderpool. Plasmids encoding Hfq $(\mathrm{pDH} 700, w t)$ and mutant derivatives (pDH701, K56A; pDH713, Y25A) are described in Ross et al [8]. Details of plasmid constructions are provided in Additional file 3 and a list of oligonucleotides used in this work is provided in Additional file 4.

Lambda phages encoding IS50 transcriptional ( $\lambda$ DBH849 and $\lambda \mathrm{DBH} 888)$ and translational $(\lambda \mathrm{DBH} 812)$ reporters were generated by cloning IS50 expression cassettes marked with 
an antibiotic resistance gene (either $k a n^{\mathrm{R}}$ or $\mathrm{cm}^{\mathrm{R}}$ ) into the his operon of pNK81 and then infecting a strain harboring one of these plasmids with $\lambda \mathrm{NK} 1039$, which also contains the his operon. Antibiotic resistant lysogens from the above crosses were selected by replica plating and subsequently phage released from the lysogens were purified, giving rise

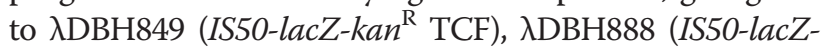
$\left.\mathrm{Cm}^{\mathrm{R}} \mathrm{TCF}\right)$ and $\lambda \mathrm{DBH} 812$ (IS50-lacZ-Kan $\left.{ }^{\mathrm{R}} \mathrm{TLF}\right)$.

E. coli strains for the 'mating out' assay were constructed by P1 transduction of Tn5 from ER2507 (NEB) into DBH33, DBH344 and DBH259. Strains containing chromosomal IS50-lacZ fusions were generated by lysogenizing DBH107 with $\lambda$ DBH849 (DBH265), $\lambda$ DBH888 (DBH303) or $\lambda$ DBH812 (DBH281). Mutant derivatives of these strains were generated by P1 transduction. A list of all of the strains, plasmids and bacteriophage used in this work is presented in Table 1.

\section{'Mating out' assay}

Conjugal 'mating out' experiments were performed essentially as described for single-copy chromosomal transposons in Ross et al. [7], except that for measuring transposition in $h f q^{-}$versus $w t$, donor growth was carried out in M9 glucose media supplemented with kanamycin $(25 \mu \mathrm{g} / \mathrm{mL})$ and amino acids, instead of LB. DBH13 was used as the recipient. Total exconjugants and transposition events with DBH179 and derivatives were scored by plating mating mixes on M9 glucose plates supplemented with leucine, thiamine and streptomycin $(150 \mu \mathrm{g} / \mathrm{mL})$ or streptomycin and kanamycin $(25 \mu \mathrm{g} / \mathrm{mL})$, respectively. Total exconjugants and transposition events with DBH261 and derivatives were scored by plating mating mixes on M9 glucose plates supplemented with leucine, thiamine, streptomycin $(150 \mu \mathrm{g} / \mathrm{mL})$ and gentamicin $(12.5 \mu \mathrm{g} / \mathrm{mL})$ or streptomycin, gentamicin and kanamycin $(25 \mu \mathrm{g} / \mathrm{mL})$, respectively.

\section{$\beta$-galactosidase assays}

Cells were grown in M9 glucose (with arginine and thiamine) or LB. In situations where strains contained plasmids, plasmids were maintained by including the appropriate antibiotic. Overnight cultures $(0.05 \mathrm{~mL})$ were used to seed subcultures $(1.5 \mathrm{~mL})$, which typically were grown to mid-log phase before being processed for the Miller assay as previously described [7].

\section{RNA isolation, primer extension and Northern blot analysis}

Total RNA was isolated essentially as described in [50]. For steady-state analysis, cells were grown to mid-log phase in LB before RNA isolation. For half-life analysis, rifampicin (dissolved in dimethyl sulfoxide; DMSO) was added to cell cultures (to $200 \mu \mathrm{g} / \mathrm{mL}$ ) to arrest transcription and RNA was isolated immediately before and after rifampicin addition at the indicated time intervals. Primer extension analysis was carried out using ${ }^{32} \mathrm{P}$-labeled primers oDH230 and oDH390, end-labeled with OptiKinase (USB, Cleveland, OH, USA) according to manufacturer's instructions. Extension reactions used $5 \mu \mathrm{g}$ of RNA, and Superscript III reverse transcriptase essentially as described in [51], except that annealing was performed at $65^{\circ} \mathrm{C}$ (with no ice treatment) before extending at $55^{\circ} \mathrm{C}$ for 45 minutes. Extension products were resolved on $6 \%$ and $10 \%$ denaturing polyacrylamide gels. For Northern blot analysis, $2 \mu \mathrm{g}$ of RNA was mixed with an equal volume of denaturing load dye $(95 \%$ deionized formamide [v/v], $10 \mathrm{mM}$ EDTA, 0.5x TBE, $3 \%$ xylene cyanol $[\mathrm{w} / \mathrm{v}]$ ), heated to $95^{\circ} \mathrm{C}$ for 2 minutes, and resolved on a $6 \%$ polyacrylamide gel containing $7 \mathrm{M}$ urea. Separated RNAs were electro-transferred to Hybond N (GE Healthcare, Mississauga, ON, Canada) in $0.5 \times \mathrm{TBE}$ and fixed with UV. Annealing and washing was performed in ULTRAhyb buffer (Ambion, Burlington, ON, Canada) according to the manufacturer's instructions, using RNA probes complimentary to SgrS or the 5S rRNA (internal standard). To construct the radiolabeled RNA probes, DNA templates for in vitro transcription were made by PCR with primers oDH232/233 (SgrS) and oDH234/235 (5S rRNA) - note that, for each primer pair, the forward primer includes the T7 core promoter. These templates were transcribed in vitro in the presence of ${ }^{32} \mathrm{P}$-UTP to generate uniformly labeled RNA probes. In vitro transcription reactions were performed in $25 \mu \mathrm{L}$ volumes with approximately $1 \mu$ g DNA template, $1 \times$ T7 RNA polymerase buffer (NEB, Beverly, MA, USA), 20 units RNasin (Promega, Madison, WI, USA), $4 \mathrm{mM}$ dithiothreitol (DTT), $0.16 \mathrm{mg} / \mathrm{mL}$ BSA, $0.4 \mathrm{mM}$ each of GTP, CTP and ATP, $0.01 \mathrm{mM}$ UTP, $50 \mu \mathrm{Ci}$ $\left[\alpha-{ }^{32} \mathrm{P}\right] \mathrm{UTP}$, and 100 units of T7 RNA polymerase.

\section{Western blot}

Cells were centrifuged ( 2 minutes at $21,000 \times g$ ), resuspended in SDS load mix (2\% [w/v] SDS, $10 \%$ [v/v] glycerol, $50 \mathrm{mM}$ Tris- $\mathrm{HCl} \mathrm{pH} \mathrm{6.8,} \mathrm{0.25 \%} \mathrm{[w/v]} \mathrm{bromophenol} \mathrm{blue,}$ $0.8 \mathrm{M} \beta$-mercaptoethanol) and heated at $95^{\circ} \mathrm{C}$ for $5 \mathrm{mi}$ nutes. To normalize for differences in growth between the various samples, the $\mathrm{OD}_{600}$ of each sample was measured and the volume spun normalized to give an equivalent to $\mathrm{OD}_{600}$ approximately equal to 0.35 . The resulting lysates were subjected to SDS-PAGE on a $12 \%$ polyacrylamide gel, proteins transferred to PVDF (Roche, Indianapolis, IN, USA) and Crp was detected by Western blot with a polyclonal rabbit anti-Crp antibody (kind gift of $\mathrm{H}$ Aiba). The primary antibody was diluted 1:20,000 in TBST; the secondary antibody (anti-rabbit IgG-horseradish peroxidase (HRP) conjugate; Promega, Madison, WI, USA) was used at 1:5,000. Crp was visualized with a Pierce ECL 2 Western blotting substrate (Thermo Scientific, Rockford, IL, USA) and PhosphorImager (GE Healthcare). The membranes were stripped and GroES detected (rabbit anti-GroES 
Table 1 Plasmids, bacteriophage and strains

\begin{tabular}{|c|c|c|c|}
\hline Strain or Plasmid & Relevant genotype & Use & Source or reference \\
\hline \multicolumn{4}{|l|}{ E. coli } \\
\hline DBH13 & 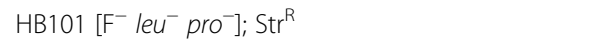 & 'Mating out' recipient & {$[36]$} \\
\hline ER2507 & zjc:: $\operatorname{Tn} 5 ; \operatorname{Kan}^{R}$ & Source of zjc:: Tn5 & NEB \\
\hline DBH179 & NK5830 [recA arg $^{-} / \mathrm{F}^{\prime}$ lacpro $\left.{ }^{+}\right]$zjc::Tn5; Kan ${ }^{\mathrm{R}}$ & 'Mating out' donor & This study \\
\hline DBH184 & DBH179 hfq-1::Qcat; Kan ${ }^{R} \mathrm{Cm}^{\mathrm{R}}$ & 'Mating out' donor & This study \\
\hline $\mathrm{DBH} 228$ & RZ211/pOX38Gen & Source of pOX38Gen & {$[37]$} \\
\hline DBH233 & HW-5 [phoA4(Am) his-45 recA1 rpsL99 met-54 F- ]; Str ${ }^{R}$ & Parent strain & {$[38]$} \\
\hline $\mathrm{DBH} 259$ & DBH233/pOX38Gen; $\operatorname{Str}^{R} G_{e n}{ }^{R}$ & Parent strain & This study \\
\hline $\mathrm{DBH} 261$ & DBH259 zjc::Tn5; Str ${ }^{R} \operatorname{Gen}^{R} \operatorname{Kan}^{R}$ & 'Mating out' donor & This study \\
\hline DBH271 & DBH261 hfq-1::Qcat; Str ${ }^{\mathrm{R}} \mathrm{Gen}^{\mathrm{R}} \mathrm{Kan}^{\mathrm{R}} \mathrm{Cm}^{\mathrm{R}}$ & 'Mating out' donor & This study \\
\hline $\mathrm{DBH} 272$ & DBH261 dam::Tn9cat; Str ${ }^{R} \operatorname{Gen}^{R} \operatorname{Kan}^{R} \mathrm{Cm}^{R}$ & 'Mating out' donor & This study \\
\hline $\mathrm{DBH} 107$ & MC4100 [F $\Delta$ (argF-lac) 169* rpsL150]; Str ${ }^{R}$ & Parent strain & [39] \\
\hline $\mathrm{DBH} 265$ & DBH107/ADBH849; $\operatorname{Str}^{R} \operatorname{Kan}^{R}$ & Miller Assay & This study \\
\hline $\mathrm{DBH} 267$ & DBH265 hfq-1::2cat; $\operatorname{Str}^{\mathrm{R}} \mathrm{Cm}^{\mathrm{R}} \mathrm{Kan}^{\mathrm{R}}$ & Miller Assay & This study \\
\hline $\mathrm{DBH} 268$ & DBH265 dam::Tn9cat; $\operatorname{Str}^{R} \mathrm{Cm}^{\mathrm{R}} \mathrm{Kan}^{\mathrm{R}}$ & Miller Assay & This study \\
\hline $\mathrm{DBH} 281$ & DBH107/ADBH812; $\operatorname{Str}^{\text {R }} \operatorname{Kan}^{R}$ & Miller Assay & This study \\
\hline $\mathrm{DBH} 283$ & DBH281 hfq-1::Qcat; $\operatorname{Str}^{\mathrm{R}} \mathrm{Cm}^{\mathrm{R}} \operatorname{Kan}^{\mathrm{R}}$ & Miller Assay & This study \\
\hline DBH285 & DBH281 dam::Tn9cat; Str ${ }^{R} \mathrm{Cm}^{R} \operatorname{Kan}^{R}$ & Miller Assay & This study \\
\hline DBH303 & DBH107/ADBH888; $\operatorname{Str}^{\mathrm{R}} \mathrm{Cm}^{\mathrm{R}}$ & Miller Assay & This study \\
\hline DBH306 & DBH303 $\Delta h f q 722:: \mathrm{kan} \mathrm{Str}^{\mathrm{R}} \mathrm{Cm}^{\mathrm{R}} \mathrm{Kan}^{\mathrm{R}}$ & Miller Assay & This study \\
\hline DBH307 & DBH303 $\Delta c r p 765:: \mathrm{kan} \mathrm{Str}^{\mathrm{R}} \mathrm{Cm}^{\mathrm{R}} \mathrm{Kan}^{\mathrm{R}}$ & Miller Assay & This study \\
\hline DBH315 & DBH303 $\Delta / r p 787:: k a n ; \operatorname{Str}^{R} \mathrm{Cm}^{\mathrm{R}} \mathrm{Kan}^{\mathrm{R}}$ & Miller Assay & This study \\
\hline DBH33 & $\mathrm{NK} 5830\left[\mathrm{recA}^{-} \mathrm{arg}^{-} / \mathrm{F}^{\prime}\right.$ lacpro $\left.{ }^{+}\right]$ & Parent strain & {$[40]$} \\
\hline $\mathrm{DBH} 16$ & DBH33 hfq-1::2cat; $\mathrm{Cm}^{\mathrm{R}}$ & Parent strain & {$[7]$} \\
\hline $\mathrm{DBH} 241$ & DBH33 dam::Tn9cat; $\mathrm{Cm}^{\mathrm{R}}$ & Parent strain & This study \\
\hline $\mathrm{DBH} 238$ & DBH33/ADBH849; $\operatorname{Kan}^{\mathrm{R}}$ & Miller Assay & This study \\
\hline DBH239 & DBH238 hfq-1::Qcat; $\operatorname{Kan}^{\mathrm{R}} \mathrm{Cm}^{\mathrm{R}}$ & Miller Assay & This study \\
\hline $\mathrm{DBH} 240$ & DBH238 dam::Tn9cat; $\operatorname{Kan}^{R} \mathrm{Cm}^{\mathrm{R}}$ & Miller Assay & This study \\
\hline DBH208 & DBH33/ADBH812; $\operatorname{Kan}^{\mathrm{R}}$ & Miller Assay & This study \\
\hline $\mathrm{DBH} 210$ & DBH208 hfq-1::Qcat; $\operatorname{Kan}^{\mathrm{R}} \mathrm{Cm}^{\mathrm{R}}$ & Miller Assay & This study \\
\hline $\mathrm{DBH} 237$ & DBH208 dam::Tn9cat; $\mathrm{Kan}^{\mathrm{R}} \mathrm{Cm}^{\mathrm{R}}$ & Miller Assay & This study \\
\hline DBH323 & DBH107 recA ${ }^{-} ; \mathrm{Str}^{\mathrm{R}}$ & Miller Assay & This study \\
\hline $\mathrm{DBH} 326$ & DBH107 recA $A^{-}$hfa-1 $\because: 2$ cat; $\operatorname{Str}^{R} \mathrm{Cm}^{\mathrm{R}}$ & Miller Assay & This study \\
\hline $\mathrm{DBH} 242$ & DBH33 $\Delta$ crp765::kan & Parent strain & This study \\
\hline $\mathrm{DBH} 344$ & $\mathrm{DBH} 242 \Delta c r p 765 ; \mathrm{Kan}^{\mathrm{S}}$ & Parent strain & This study \\
\hline $\mathrm{DBH} 345$ & DBH344 zjc::Tn5; $\mathrm{Kan}^{R}$ & 'Mating out' donor & This study \\
\hline TM338 & W3110m/c rne-Flag-cat; rif $^{S} \mathrm{Cm}^{\mathrm{R}}$ & RNA half-life measurements & [41] \\
\hline TM618 & W3110m/c rne-Flag-cat $\Delta h f q ;$ rif $^{S} \mathrm{Cm}^{\mathrm{R}}$ & RNA half-life measurements & {$[42]$} \\
\hline $\mathrm{DH} 5 \mathrm{a}$ & $\operatorname{rec} A^{-}$ & Plasmid propagation & Invitrogen \\
\hline \multicolumn{4}{|l|}{ Plasmids } \\
\hline pWKS30 & pSC101-derived; low copy-number ori ; $A p^{R}$ & 'Empty vector' for Hfq expression & {$[35]$} \\
\hline pDH700 & pWKS30-P3-hfawi Ap ${ }^{R}$ & $\mathrm{Hfq}_{W T}$ expression & [7] \\
\hline pDH701 & pWKS30-P3-hfq $q_{K 56 A ;} A^{R}$ & $\mathrm{Hfq}_{\mathrm{K} 56 \mathrm{~A}}$ expression & [7] \\
\hline pDH713 & pWKS30-P3-hfa $q_{Y 25 A} ; A^{R}$ & $\mathrm{Hfq}_{\mathrm{Y} 25 \mathrm{~A}}$ expression & [8] \\
\hline
\end{tabular}


Table 1 Plasmids, bacteriophage and strains (Continued)

\begin{tabular}{|c|c|c|c|}
\hline pDH533 & pUC18-derivative; Tn5 t'ase M56A; $\mathrm{Ap}^{\mathrm{R}} \mathrm{Cm}^{\mathrm{R}}$ & Source of $\operatorname{Tn} 5$ transposase (No $\operatorname{lnh})$. & [43] \\
\hline pDH752 & pDH533 with t'ase mutated to $G 53 \mathrm{~A}, \mathrm{C} 61 \mathrm{~A} ; \mathrm{Ap}^{\mathrm{R}} \mathrm{Cm}^{\mathrm{R}}$ & DAM-insensitive t'ase & This study \\
\hline pDH828 & pDH533 with t'ase mutated to D97A; $\mathrm{Ap}^{\mathrm{R}} \mathrm{Cm}^{\mathrm{R}}$ & Catalytic ${ }^{-}$t'ase $^{\prime}$ & This study \\
\hline pNK81 & pBR333-derivative; encodes his operon; $A p^{R}$ & Lambda crosses & [44] \\
\hline pDH682 & pUC18-derivative; IS50-lacZ TCF; Ap ${ }^{R}$ & Source of TCF & This study \\
\hline pDH838 & pDH682-derivative; TCF 'marked' with $\mathrm{kan}^{\mathrm{R}}$ & Parent of $\mathrm{pDH} 849$ & This study \\
\hline pDH883 & pDH682-derivative; TCF 'marked' with $\mathrm{cm}^{\mathrm{R}}$ & Parent of pDH888 & This study \\
\hline pDH849 & TCF-kan ${ }^{R}$ from pDH682 cloned into Bcll-cut pNK81; pp $^{R} K{ }^{R}$ & For crossing TCF onto $\lambda$ & This study \\
\hline pDH888 & TCF-cm ${ }^{R}$ cloned onto Bcll-cut pNK81; $\mathrm{Ap}^{\mathrm{R}} \mathrm{Cm}^{\mathrm{R}}$ & For crossing TCF onto $\lambda$ & This study \\
\hline pDH658 & pRZ9905-derivative; full-length IS50-lacZ TLF; Ap ${ }^{R}$ & Parent of pDH795 & This study \\
\hline pDH795 & pDH658-derivative; 'deletion' TLF used in this study; $A p^{R}$ & Parent of pDH804 & This study \\
\hline pDH804 & pDH795-derivative; TLF 'marked' with $\operatorname{kan}^{\mathrm{R}}$ & Parent of pDH812 & This study \\
\hline pDH812 & TLF-kan ${ }^{R}$ cloned into Bcll-cut pNK81; Ap ${ }^{R} \operatorname{Kan}^{R}$ & For crossing TLF onto $\lambda$ & This study \\
\hline pDH753 & pWKS30-derivative; contains IS50-lacZ TLF from pDH658; Ap $^{R}$ & Parent of pDH798 & This study \\
\hline pDH798 & pDH753-derivative; $\mathrm{Ap}^{\mathrm{S}} \mathrm{Kan}^{\mathrm{R}}$ & Miller Assay & This study \\
\hline pDH763 & pBR-plac; $A p^{R}$ & Vector for sRNA-induction & [45] \\
\hline pDH764 & pBR-plac-sgrs; $A p^{R}$ & SgrS-induction & [46] \\
\hline pDH895 & pBR-plac-sgrS1; $A p^{R}$ & SgrS1-induction & [47] \\
\hline pDH766 & pBR-plac-rybB; $A p^{R}$ & RybB-induction & [48] \\
\hline $\mathrm{pDH} 768$ & pBR-plac-micC; $\mathrm{Ap}^{\mathrm{R}}$ & MicC-induction & [48] \\
\hline pDH772 & pBR-plac-ryeB; $A^{R}$ & RyeB-induction & [48] \\
\hline pDH908 & pDH795-derivative; Lpp-TLF & Miller Assay & This study \\
\hline \multicolumn{4}{|l|}{ Phage } \\
\hline$\lambda N K 1039$ & Encodes his operon & Parent phage & [49] \\
\hline$\lambda \mathrm{DBH} 812$ & IS50-lacZ translational fusion (TLF) from pDH812 marked with kan $^{R}$ & Chromosomal TLF construction & This study \\
\hline$\lambda \mathrm{DBH} 849$ & IS50-lacZ transcriptional fusion (TCF) marked with kan ${ }^{R}$ & Chromosomal TCF construction & This study \\
\hline$\lambda \mathrm{DBH} 888$ & IS50-lacZ transcriptional fusion (TCF) marked with $\mathrm{cm}^{\mathrm{R}}$ & Chromosomal TCF construction & This study \\
\hline
\end{tabular}

antibody from Sigma-Aldrich (St Louis, MO, USA) at $1: 10,000)$ for use as an internal standard; GroES is not sensitive to $h f q$ status [19]. Bands were quantified using ImageQuant software (GE Healthcare) and Crp levels plotted relative to GroES.

\section{Additional files}

Additional file 1: Mapping Tn5 transposition events. Southern blot and ST-PCR characterization of Tn5 transposition events in wt and $\mathrm{hfq}^{-}$strains.

Additional file 2: Impact of SgrS over-expression on growth rate in M9 glucose. Growth curves of cells in which SgrS RNA was or was not induced by IPTG addition and corresponding Northern blot showing SgrS levels.

Additional file 3: Details of plasmids constructed for this work. Additional file 4: List of oligonucleotides used in this work.

\section{Abbreviations}

BSA: bovine serum albumin; Crp: cyclic AMP-receptor protein; DAM: DNA adenine methylase; DMSO: dimethyl sulfoxide; DTT: dithiothreitol; EDTA: ethylenediaminetetraacetic acid; LB: Luria broth; PAGE: polyacrylamide gel electrophoresis; PCR: polymerase chain reaction; RT-PCR: reverse transcription polymerase chain reaction; sRNA: small RNA; SDS: sodium dodecyl sulfate; TBE: Tris borate-EDTA buffer; TBST: 20 mM Tris-HCl (pH 7.5), 150 mM sodium chloride, 0.5\% Tween-20; TCF: transcriptional fusion; TIR: translation initiation region; TLF: translational fusion; UTR: untranslated region; UV: ultraviolet light.

\section{Competing interests}

The authors declare that they have no competing interest.

\section{Authors' contributions}

JR performed 'mating out' assays, $\beta$-galactosidase assays in Figures 3, 7 and 8, RT-PCR assays, Western blot assays, participated in the design of the study and helped draft the manuscript. RT performed the $\beta$-galactosidase assay in Figure 4, steady-state transcript measurements and RNA half-life measurements and prepared figures. MDB helped construct chromosomal reporter strains. CRM made the initial discovery that $\operatorname{Tn} 5$ transposition is up-regulated under conditions of hfq deficiency and constructed IS50-reporter plasmids and expression plasmids. DBH participated in the design of the study, helped in constructing strains and plasmids, performed the 'mating out' experiment in Figure 7D and drafted the manuscript. All authors read and approved the final manuscript.

\section{Acknowledgements}

We thank Michael Ellis for providing comments on the manuscript and for useful discussions, and Claire Young for assistance with P1 transductions. We also thank S Gottesman and C Vanderpool for providing pLlacO-sRNA 
expression plasmids and the sgrs1 allele, respectively, and $\mathrm{H}$ Aiba for providing an anti-Crp antibody. Finally, we thank W Reznikoff for providing IS50 transposase plasmids and T Naas for providing an E. coli strain harboring pOX38-Gen. This work was supported by a grant to DBH (MOP 11281) from Canadian Institutes of Health Research. JR and CM were supported by OGS and NSERC scholarships.

Received: 18 August 2014 Accepted: 11 November 2014 Published online: 30 November 2014

\section{References}

1. Roberts D, Hoopes BC, McClure WR, Kleckner N: IS10 transposition is regulated by DNA adenine methylation. Cell 1985, 43(1):117-130

2. Yin JC, Krebs MP, Reznikoff WS: Effect of dam methylation on Tn5 transposition. J Mol Bio/ 1988, 199(1):35-45.

3. Raleigh EA, Kleckner N: Quantitation of insertion sequence IS10 transposase gene expression by a method generally applicable to any rarely expressed gene. Proc Natl Acad Sci U S A 1986, 83(6):1787-1791.

4. Krebs MP, Reznikoff WS: Transcriptional and translational initiation sites of IS50. Control of transposase and inhibitor expression. J Mol Biol 1986, 192(4):781-791.

5. Simons RW, Kleckner N: Translational control of IS10 transposition. Cell 1983, 34(2):683-691.

6. Ma C, Simons RW: The IS10 antisense RNA blocks ribosome binding at the transposase translation initiation site. EMBO J 1990, 9(4):1267-1274.

7. Ross JA, Wardle SJ, Haniford DB: Tn10/IS10 transposition is downregulated at the level of transposase expression by the RNA-binding protein $\mathrm{Hfq}$. Mol Microbiol 2010, 78(3):607-621.

8. Ross JA, Ellis MJ, Hossain S, Haniford DB: Hfq restructures RNA-IN and RNA-OUT and facilitates antisense pairing in the Tn10/IS10 system. RNA 2013, 19(5):670-684

9. Vogel J, Luisi BF: Hfq and its constellation of RNA. Nat Rev Microbio/ 2011, 9(8):578-589.

10. Le Derout J, Boni IV, Regnier P, Hajnsdorf E: Hfq affects mRNA levels independently of degradation. BMC Mol Biol 2010, 11:17

11. Mahillon J, Chandler M: Insertion sequences. Microbiol Mol Biol Rev 1998, 62(3):725-774

12. Kennedy AK, Guhathakurta A, Kleckner N, Haniford DB: Tn10 transposition via a DNA hairpin intermediate. Cell 1998, 95(1):125-134

13. Bhasin A, Goryshin IY, Reznikoff WS: Hairpin formation in Tn5 transposition. J Biol Chem 1999, 274(52):37021-37029.

14. Mahnke Braam LA, Goryshin IY, Reznikoff WS: A mechanism for Tn5 inhibition. carboxyl-terminal dimerization. J Biol Chem 1999, 274(1):86-92.

15. Reznikoff WS: Transposon Tn5. Annu Rev Genet 2008, 42:269-286.

16. Kuan CT, Tessman I: LexA protein of Escherichia coli represses expression of the Tn5 transposase gene. J Bacterio/ 1991, 173(20):6406-6410.

17. Mikulecky PJ, Kaw MK, Brescia CC, Takach JC, Sledjeski DD, Feig AL: Escherichia coli $\mathrm{Hfq}$ has distinct interaction surfaces for DsrA, rpoS and poly(A) RNAs. Nat Struct Mol Biol 2004, 11(12):1206-1214.

18. Martinez-Antonio A, Collado-Vides J: Identifying global regulators in transcriptional regulatory networks in bacteria. Curr Opin Microbiol 2003, 6(5):482-489.

19. Guisbert E, Rhodius VA, Ahuja N, Witkin E, Gross CA: Hfq modulates the sigmaE-mediated envelope stress response and the sigma32-mediated cytoplasmic stress response in Escherichia coli. J Bacteriol 2007. 189(5):1963-1973.

20. Lathem WW, Schroeder JA, Bellows LE, Ritzert JT, Koo JT, Price PA, Caulfield AJ, Goldman WE: Posttranscriptional regulation of the Yersinia pestis cyclic AMP receptor protein Crp and impact on virulence. mBio 2014, 5(1):e01038-01013.

21. Hussein R, Lim HN: Disruption of small RNA signaling caused by competition for Hfq. Proc Natl Acad Sci U S A 2011, 108(3):1110-1115.

22. Moon K, Gottesman S: Competition among Hfq-binding small RNAs in Escherichia coli. Mol Microbiol 2011, 82(6):1545-1562.

23. Wassarman KM, Repoila F, Rosenow C, Storz G, Gottesman S: Identification of novel small RNAs using comparative genomics and microarrays. Genes Dev 2001, 15(13):1637-1651.

24. Ishikawa H, Otaka H, Maki K, Morita T, Aiba H: The functional Hfq-binding module of bacterial sRNAs consists of a double or single hairpin preceded by a U-rich sequence and followed by a $3^{\prime}$ poly(U) tail. RNA (New York, NY) 2012, 18(5):1062-1074.

25. Fender A, Elf J, Hampel K, Zimmermann B, Wagner EG: RNAs actively cycle on the Sm-like protein Hfq. Genes Dev 2010, 24(23):2621-2626.

26. Papenfort K, Vogel J: Small RNA functions in carbon metabolism and virulence of enteric pathogens. Front Cell Infect Microbiol 2014, 4:91.

27. Agre $\mathrm{P}$, Bonhivers $\mathrm{M}$, Borgnia MJ: The aquaporins, blueprints for cellular plumbing systems. J Bio/ Chem 1998, 273(24):14659-14662.

28. Rice JB, Vanderpool CK: The small RNA SgrS controls sugar-phosphate accumulation by regulating multiple PTS genes. Nucleic Acids Res 2011 39(9):3806-3819.

29. Hu ST, Wang HC, Lei GS, Wang SH: Negative regulation of IS2 transposition by the cyclic AMP (cAMP)-cAMP receptor protein complex. J Bacteriol 1998, 180(10):2682-2688

30. Grainger DC, Hurd D, Harrison M, Holdstock J, Busby SJ: Studies of the distribution of Escherichia coli CAMP-receptor protein and RNA polymerase along the E. coli chromosome. Proc Natl Acad Sci U S A 2005, 102(49):17693-17698.

31. Johansen J, Eriksen M, Kallipolitis B, Valentin-Hansen P: Down-regulation of outer membrane proteins by noncoding RNAs: unraveling the CAMPCRP- and sigmaE-dependent CyaR-ompX regulatory case. J Mol Biol 2008, 383(1):1-9.

32. Frohlich $K S$, Papenfort $K$, Berger AA, Vogel J: A conserved RpoS-dependent small RNA controls the synthesis of major porin OmpD. Nucleic Acids Res 2012, 40(8):3623-3640.

33. Chen S, Zhang A, Blyn LB, Storz G: MicC, a second small-RNA regulator of Omp protein expression in Escherichia coli. J Bacteriol 2004, 186(20):6689-6697.

34. Twiss E, Coros AM, Tavakoli NP, Derbyshire KM: Transposition is modulated by a diverse set of host factors in Escherichia coli and is stimulated by nutritional stress. Mol Microbiol 2005, 57(6):1593-1607.

35. Wang RF, Kushner SR: Construction of versatile low-copy number vectors for cloning, sequencing and gene expression in Escherichia coli. Gene 1991, 100:195-199.

36. Bolivar F, Backman K: Plasmids of Escherichia coli as cloning vectors. Methods Enzymol 1979, 68:245-267.

37. Cuzon G, Naas T, Nordmann P: Functional characterization of Tn4401, a Tn3-based transposon involved in blaKPC gene mobilization. Antimicrob Agents Chemother 2011, 55(11):5370-5373.

38. Hoffman EP, Wilhelm RC: Genetic mapping and dominance of the amber suppressor, Su1 (supD), in Escherichia coli K-12. J Bacteriol 1970, 103(1):32-36.

39. Casadaban MJ: Transposition and fusion of the lac genes to selected promoters in Escherichia coli using bacteriophage lambda and $\mathrm{Mu}$. J Mol Biol 1976, 104(3):541-555.

40. Foster TJ, Davis MA, Roberts DE, Takeshita K, Kleckner N: Genetic organization of transposon Tn10. Cell 1981, 23(1):201-213.

41. Morita T, Kawamoto H, Mizota T, Inada T, Aiba H: Enolase in the RNA degradosome plays a crucial role in the rapid decay of glucose transporter mRNA in the response to phosphosugar stress in Escherichia coli. Mol Microbiol 2004, 54(4):1063-1075.

42. Morita T, Maki K, Aiba H: RNase E-based ribonucleoprotein complexes: mechanical basis of mRNA destabilization mediated by bacterial noncoding RNAs. Genes Dev 2005, 19(18):2176-2186.

43. Whitfield CR, Wardle SJ, Haniford DB: The global bacterial regulator H-NS promotes transpososome formation and transposition in the Tn5 system. Nucleic Acids Res 2009, 37(2):309-321.

44. Way JC, Davis MA, Morisato D, Roberts DE, Kleckner N: New Tn10 derivatives for transposon mutagenesis and for construction of lacZ operon fusions by transposition. Gene 1984, 32(3):369-379.

45. Guillier M, Gottesman S: Remodelling of the Escherichia coli outer membrane by two small regulatory RNAs. Mol Microbiol 2006, 59(1):231-247.

46. Vanderpool CK, Gottesman S: Involvement of a novel transcriptional activator and small RNA in post-transcriptional regulation of the glucose phosphoenolpyruvate phosphotransferase system. Mol Microbiol 2004 54(4):1076-1089.

47. Wadler CS, Vanderpool CK: Characterization of homologs of the small RNA SgrS reveals diversity in function. Nucleic Acids Res 2009, 37(16):5477-5485.

48. Mandin P, Gottesman S: Integrating anaerobic/aerobic sensing and the general stress response through the ArcZ small RNA. EMBO J 2010, 29(18):3094-3107.

49. Haniford DB, Chelouche AR, Kleckner N: A specific class of IS10 transposase mutants are blocked for target site interactions and 
promote formation of an excised transposon fragment. Cell 1989, 59(2):385-394.

50. Garrey SM, Mackie GA: Roles of the 5'-phosphate sensor domain in RNase E. Mol Microbiol 2011, 80(6):1613-1624.

51. Wilkinson KA, Merino EJ, Weeks KM: Selective 2'-hydroxyl acylation analyzed by primer extension (SHAPE): quantitative RNA structure analysis at single nucleotide resolution. Nat Protoc 2006, 1(3):1610-1616.

doi:10.1186/s13100-014-0027-z

Cite this article as: Ross et al: Tn5 transposition in Escherichia coli is repressed by $\mathrm{Hfq}$ and activated by over-expression of the small non-coding RNA SgrS. Mobile DNA 2014 5:27.

\section{Submit your next manuscript to BioMed Central and take full advantage of:}

- Convenient online submission

- Thorough peer review

- No space constraints or color figure charges

- Immediate publication on acceptance

- Inclusion in PubMed, CAS, Scopus and Google Scholar

- Research which is freely available for redistribution 